\title{
Competition between compaction of single chains and bundling of multiple chains in giant DNA molecules
}

\section{$\operatorname{AUTHOR}(\mathrm{S})$ :}

Iwataki, T; Kidoaki, S; Sakaue, T; Yoshikawa, K; Abramchuk, SS

\section{CITATION:}

Iwataki, T ... [et al]. Competition between compaction of single chains and bundling of multiple chains in giant DNA molecules. JOURNAL OF CHEMICAL PHYSICS 2004, 120(8): 4004-4011

\section{ISSUE DATE:}

2004-02-22

URL:

http://hdl.handle.net/2433/49855

\section{RIGHT:}

Copyright 2004 American Institute of Physics. This article may be downloaded for personal use only. Any other use requires prior permission of the author and the American Institute of Physics. 


\title{
Competition between compaction of single chains and bundling of multiple chains in giant DNA molecules
}

\author{
Toshio Iwataki, ${ }^{\text {a) }}$ Satoru Kidoaki, ${ }^{\text {b) }}$ Takahiro Sakaue, Kenichi Yoshikawa, ${ }^{c)}$ \\ and Sergey S. Abramchuk ${ }^{\text {d) }}$ \\ Department of Physics, Graduate School of Science, Kyoto University and CREST (Core Research for \\ Evolutional Science and Technology) of Japan Sciences and Technology Corporation, Kyoto 606-8502, Japan
}

(Received 8 September 2003; accepted 25 November 2003)

\begin{abstract}
It has been established that in a dilute solution individual giant DNA molecules undergo a large discrete transition between an elongated coil state and a folded compact state. On the other hand, in concentrated solutions, DNA molecules assemble into various characteristic states, including multichain aggregate, liquid crystalline, ionic crystal, etc. In this study, we compared single-chain and multiple-chain events by observing individual chains using fluorescence microscopy. We used spermidine, $\operatorname{SPD}(3+)$, as a condensing agent for giant DNA. When the concentration of DNA is below $1 \mu \mathrm{M}$ in base-pair units, individual DNA molecules exhibit a transition from an elongated state to a compact state. When the concentration of DNA is increased to $10 \mu \mathrm{M}$, a thick fiberlike assembly of multiple chains appears. AFM measurements of this thick fiber revealed that more than tens of DNA molecules form a bundle structure with parallel ordering of the chains. The transition between single-chain compaction and bundle formation with multiple-chain assemblies was reproduced by a theoretical calculation. (C) 2004 American Institute of Physics.
\end{abstract}

[DOI: $10.1063 / 1.1642610]$

\section{INTRODUCTION}

In the living cellular environment, long DNA molecules on the order of mega base pairs, or with lengths in the millimeter to centimeter range, are folded into a compact state on the order of micrometers. On the other hand, it is well known that such long DNAs dissolve in usual aqueous solution with an elongated coil conformation, i.e., water is a good solvent for DNA. ${ }^{1,2}$ In the usual aqueous environment as a good solvent, the persistence length of DNA is around $50 \mathrm{~nm}$ (corresponding to 130 base pairs), ${ }^{3}$ suggesting that short oligomeric DNA molecules behave as rigid straight rods. Thus condensing agents induce aggregation and/or precipitation in such short DNAs, without single-chain compaction. On the other hand, it has become clear that giant DNA molecules undergo a large discrete transition from an elongated coil state into a folded compact state. ${ }^{4-6}$ Compact DNA's behave as a soluble colloidal particle smaller than 0.1 $\mu \mathrm{m}$, where the negative charge almost disappears within the volume of the particle. ${ }^{7}$

DNA is a stiff polymer, and the persistence length (approximately $50 \mathrm{~nm}$ ) is much larger than the thickness (approximately $2 \mathrm{~nm}$ ) of the double-stranded structure. According to textbooks of macromolecular science, the end-to-end distance $R$ of an elongated polymer chain can be written as

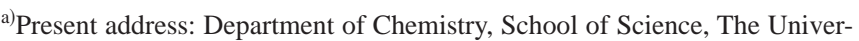
sity of Tokyo, Tokyo, 113-0033, Japan.

b) Present address: Department of Biomedical Engineering, Graduate School of Medicine, Kyushu University, Fukuoka, 812-8582, Japan.

c) Author to whom correspondence should be addressed. Electronic mail: yoshikaw@scphys.kyoto-u.ac.jp

d)Permanent address: Institute of Organoelement Compounds, Russian Academy of Sciences, Vavilova St. 28. Moscow 117813, Russia.
}

$$
R_{\text {coil }} \approx l_{K} \cdot N^{3 / 5},
$$

where the length of a Kuhn segment $l_{K} \approx 2 \lambda$ ( $\lambda$ is the persistence length) and $N$ is the number of segments. Thus the volume of a single giant DNA is given as

$$
V_{\text {coil }} \sim R_{\text {coil }}^{3} \sim l_{K}^{3} N^{9 / 5} \text {. }
$$

We take the area of cross reaction in double-stranded DNA as $s ; s=(1 / 4) \pi d^{2}$ where $d$ is the diameter (around $2 \mathrm{~nm}$ ).

The volume of a single chain in the compact state is given as

$$
V_{\text {comp }} \approx l_{K} \cdot s \cdot N .
$$

If we tentatively consider a giant DNA of $30 k$ base pairs, the volume ratio of the coiled and compact states becomes

$$
V_{\text {coil }} / V_{\text {comp. }} \sim 10^{5} \text {. }
$$

This means that the density of the segments changes by five orders of magnitude with the folding transition in a single chain. In fact, such a large change in density has been confirmed through the observation of individual giant DNA molecules using fluorescence microscopy. ${ }^{4}$ In the present study, we focused on the next important question; i.e., what will happen with multiple chains of such giant DNAs?

Several studies have been performed to clarify the structure and mechanism of "DNA condensation." As indicated by Bloomfield ${ }^{8}$ the term "DNA condensation" has been mostly used to describe multiple-chain assembly. In fact, various highly ordered states have been reported in relation to "DNA condensation," including the formation of liquid crystals. ${ }^{9-12}$ It is well known that a self-avoiding volume effect, or repulsive interaction, is essential for the stability of liquid crystalline. ${ }^{13}$ On the other hand, the compaction of 
single DNA is the result of effective attraction between segments within a molecular chain. Thus it would be interesting to study the "condensation" of both single and multiple chains with an increase in the polymer concentration, while maintaining the attraction between the polymer segments.

We used spermidine as a condensing agent for giant DNA. Multivalent polyamines such as spermidine or spermine are generally present in living cells, and the interaction of polyamines with DNA has been extensively studied both by experiments ${ }^{9-12,14-17}$ and simulations. ${ }^{18-21}$

\section{EXPERIMENTAL SECTION}

\section{Materials}

T4 bacteriophage DNA (166k base pairs) was purchased from Sigma Chemicals (St. Louis, MO). To visualize DNA molecules, we used a fluorescent dye, 4', 6-diamidino-2phenylindole (DAPI, Wako Pure Chemical, Osaka, Japan), since it has minimal effects on the conformational dynamics of giant DNA molecules. ${ }^{22}$ 2-Mercaptoethanol (2-ME) and spermidine trihydrochloride $[\operatorname{SPD}(3+)]$ were purchased from Nacalai Tesque (Kyoto, Japan). 2-ME was used as a free-radical scavenger to reduce fluorescent fading and lightinduced damage of DNA.

\section{Fluorescence microscopic measurements}

As a host polymer solution, the desired amount of spermidine was added to Tris- $\mathrm{HCl}$ buffer $(10 \mathrm{mM}$ Tris, $p \mathrm{H} \mathrm{8.0)}$ containing DAPI and 2-ME. After vortexing, T4 DNA solution was mixed to give the final concentrations of $0.1 \mu \mathrm{M}$ DAPI and $4 \%(\mathrm{v} / \mathrm{v}) 2-\mathrm{ME}$. It has been confirmed ${ }^{3}$ that the persistence length and contour length of DNA remain constant under such a low DAPI concentration. Fluorescence microscopic observations were performed after $1 \mathrm{~h}$ of equilibration. Sample solution was situated between two thin glass plates separated by approximately $150 \mu \mathrm{m}$ using spacer glass. Fluorescent images were observed with a Carl Zeiss Axiovert 135 TV microscope and recorded through a Hamamatsu SIT TV camera and an image processor (Argus 10, Hamamatsu Photonics).

\section{AFM observation}

To measure the thickness of DNA fibers, we used an atomic force microscope (AFM) equipped with a fluorescence microscope (NBV100, Olympus Optical Co., Ltd., Tokyo, Japan; AFM controller and software: Nanoscope IIIa, Digital Instrument, CA; FM: IX70, Olympus). Thirty microliters of the sample solution (T4 DNA: $10 \mu \mathrm{M}$ (in base pairs), spermidine: $300 \mu \mathrm{M}$, DAPI: $0.1 \mu \mathrm{M}, 2-\mathrm{ME}$ : $4 \%(\mathrm{v} /$ $\mathrm{v})$, in Tris- $\mathrm{HCl}$ buffer $[p \mathrm{H} 8.0]$ ) were dropped on a glass cover slide and allowed to sit for $10 \mathrm{~min}$ to adsorb the fibers. Excess solution on the glass plate was gently blown off with a jet of nitrogen gas, and dried under air. The glass cover slide was then subjected to $10 \mathrm{~min}$ of sonication in ethanol, rinsed with distilled water, and dried under air. Adsorbed fibers on the glass plate were searched for by fluorescence microscope and any fibers found were observed by tappingmode AFM under air using a silicon single-crystal cantilever
(A)
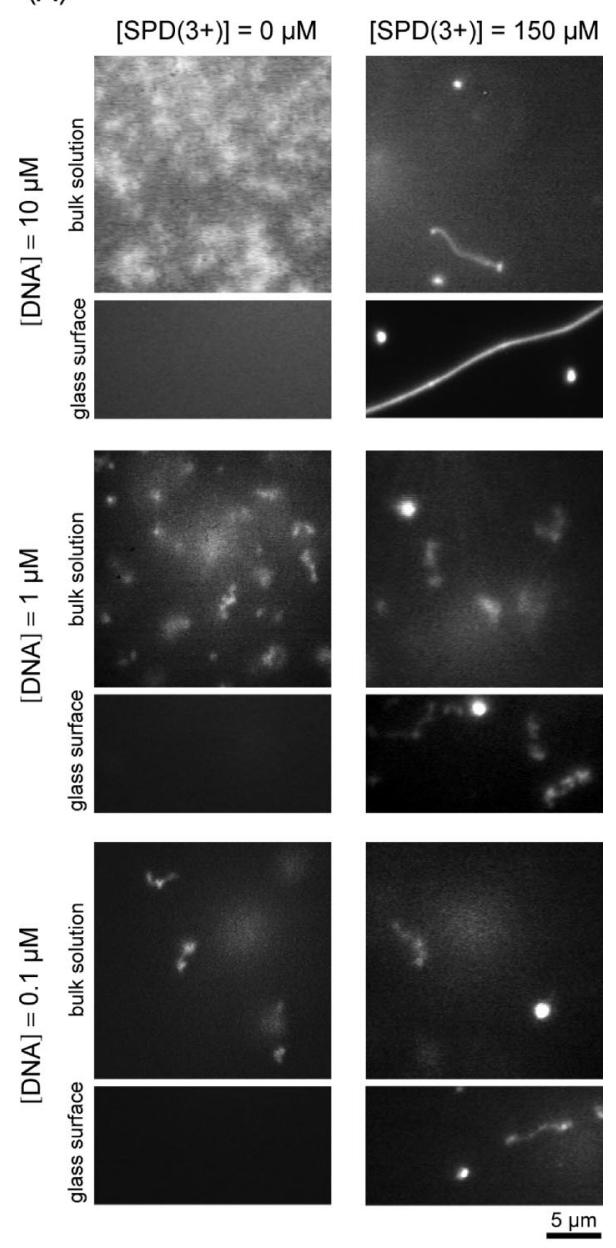

(B)

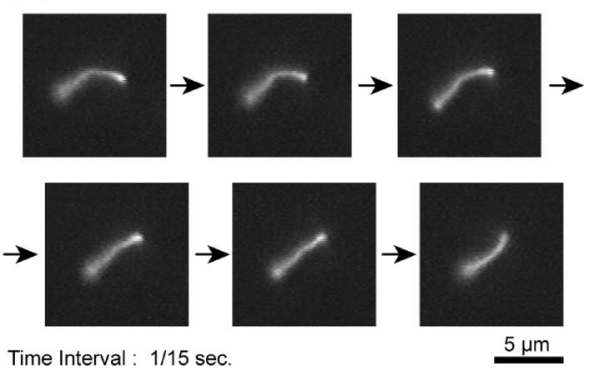

FIG. 1. (a) Fluorescence images of stained T4 DNA at various DNA concentrations, without (left) and with (right) $150 \mu \mathrm{M}$ spermidine, $\operatorname{SPD}(3+)$. DNAs are scarcely adsorbed on the glass surface in the absence of $\operatorname{SPD}(3+)$. (b) Brownian motion of a thick bundle in bulk solution at $10 \mu \mathrm{M}$ T4 DNA and $150 \mu \mathrm{M} \operatorname{SPD}(3+)$, indicating that the bundles exhibit a semiflexible nature.

(OMCL-AC120TS-1, Olympus). The free vibrating amplitude was set at about $1.5 \mathrm{~V}$ and the setpoint was taken as 0.9 V.

\section{RESULTS}

Figure 1(a) shows fluorescence microscopic images of T4 DNA molecules stained by the fluorescent dye DAPI. The nature of the transition under a low DNA concentration $[\mathrm{DNA}]=0.1 \mu \mathrm{M}$ in base pair units is essentially the same as in previous studies. ${ }^{3-6}$ At low DNA concentrations, indi- 


\section{Concentrated DNA solution $(>10 \mu \mathrm{M})$}
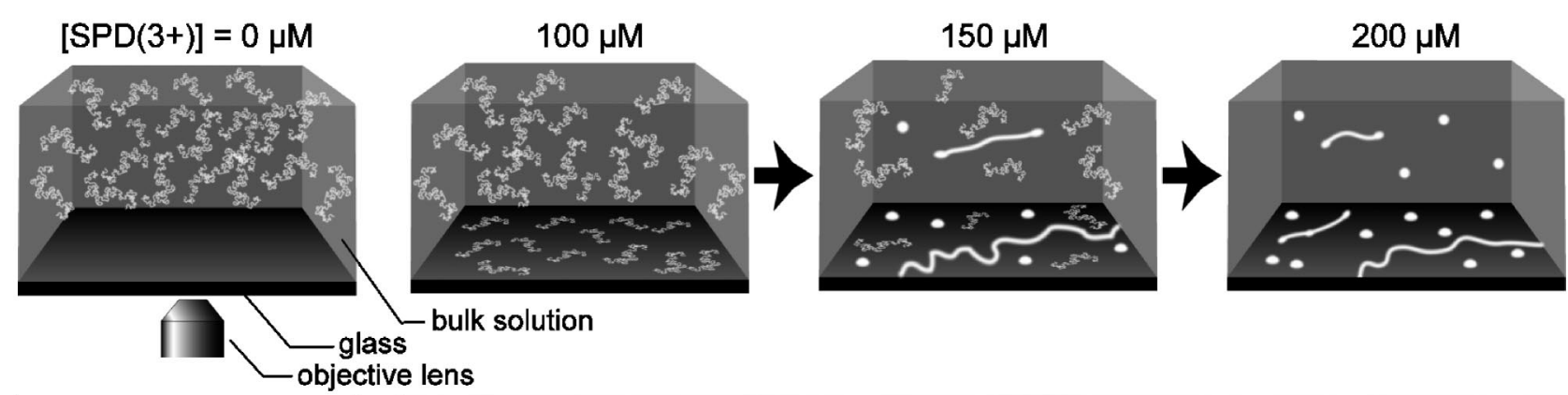

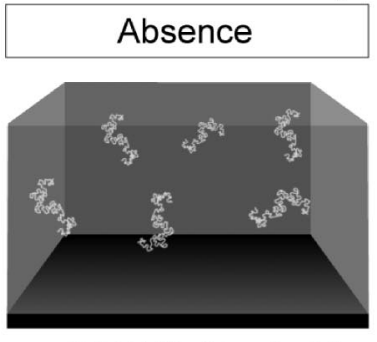

$[\mathrm{SPD}(3+)]=0 \mu \mathrm{M}$

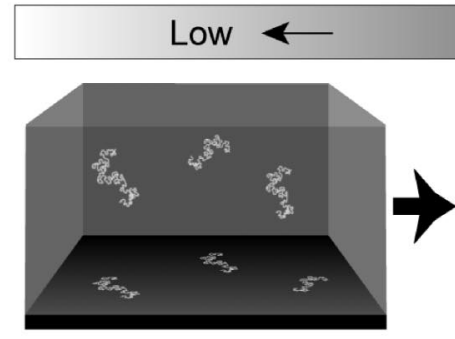

$100 \mu \mathrm{M}$ [condensing agent]

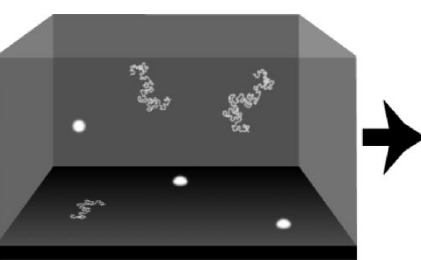

$150 \mu \mathrm{M}$
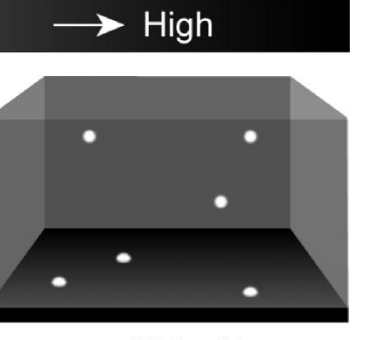

$200 \mu \mathrm{M}$

\section{Diluted DNA solution $(<1 \mu \mathrm{M})$}

FIG. 2. Schematic representation of the conformational change in DNA molecules with an increase in the SPD $(3+)$ concentration. In dilute solution, individual DNAs show an all-or-none folding transition with the addition of $\operatorname{SPD}(3+)$. In contrast, in concentrated solution, both single-chain compaction and multiple-chain bundling are seen.

vidual DNA molecules undergo a large discrete transition from an elongated coil state to a folded compact state with an increase in the concentration of condensing agent. At $[\mathrm{DNA}]=1 \mu \mathrm{M}$, the nature of the structural transition is again essentially the same: the elongated and compact states appear to coexist at $[\operatorname{SPD}(3+)]=150 \mu \mathrm{M}$. When the DNA concentration is increased to $10 \mu \mathrm{M}$, a thick bundle composed of multiple DNA molecules is found, under a spermidine concentration similar to that used to induce the folding transition from coiled to compact states in dilute DNA solutions. At $[\mathrm{DNA}]=10 \mu \mathrm{M}$ and $[\operatorname{SPD}(3+)]=0 \mu \mathrm{M}$, the blurred faint image on the left corresponds to an elongated DNA, which can be recognized by visual observation of the changing image. The figure also shows images on the glass surface, and indicates that no DNA molecules are absorbed on the surface in the absence of $\operatorname{SPD}(3+)$. Figure 1(b) shows Brownian motion of a thick bundle formed at a DNA concentration of $10 \mu \mathrm{M}$ in the presence of $150 \mu \mathrm{M}$ spermidine. Under this condition, the length of the bundle is distributed between several $\mu \mathrm{m}$ and several hundred $\mu \mathrm{m}$. The structure of the bundle exhibits thermal fluctuation, suggesting that the bundle is not so stiff but rather semiflexible. Thus the packing of DNA is expected to be slightly swollen.

Figure 2 shows a schematic representation of the nature of the structural transition in T4 DNA molecules induced by an increase in the spermidine concentration. At low DNA concentrations, the bulk solution mostly contains elongated DNA molecules when the spermidine concentration is below $100 \mu \mathrm{M}$. When $[\operatorname{SPD}(3+)]$ is increased to $150 \mu \mathrm{M}$, the elongated coil and folded compact states coexist in the bulk solution and also on the glass surface. When $[\operatorname{SPD}(3+)]$ is increased to $200 \mu \mathrm{M}$, all of the DNA molecules assume the folded compact state. Through the visual observation of individual DNA molecules, we confirmed that the conformation on the surface is essentially the same as that in the bulk solution, as is schematically represented in the lower part of Fig. 2. On the other hand, at a higher DNA concentration, thick bundles are formed together with folded compact globules. The thick bundles remain in the bulk solution, as long as they are shorter than a few tens of $\mu \mathrm{m}$, whereas long bundles tend to accumulate on the glass surface. The soluble nature of the short bundles, as well as the globules from single DNA molecules, indicate a colloidal character; i.e., the surfaces of the bundle and globule are highly charged.

Figure 3 shows a diagram of the state as a function of the DNA concentration and $[\operatorname{SPD}(3+)]$. Regardless of the DNA concentration, the structural transitions exhibit similar
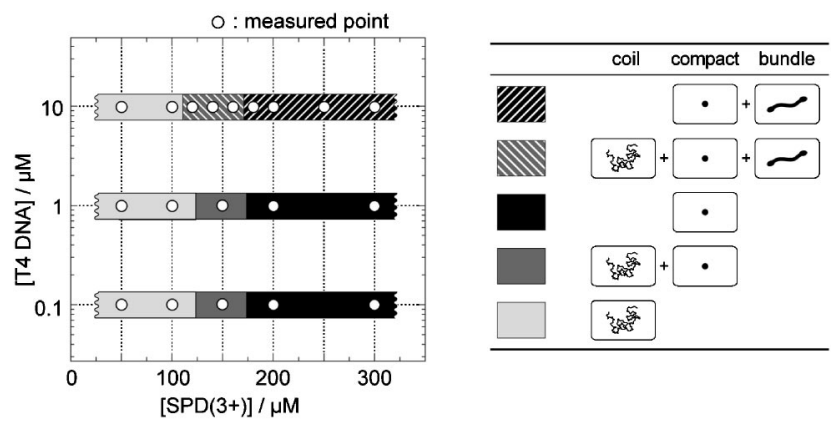

FIG. 3. Diagram of the dissolution state as a function of [T4 DNA] vs $[\operatorname{SPD}(3+)]$. The concentration of spermidine needed to condense DNAs remains nearly constant regardless of the concentration of DNA. 


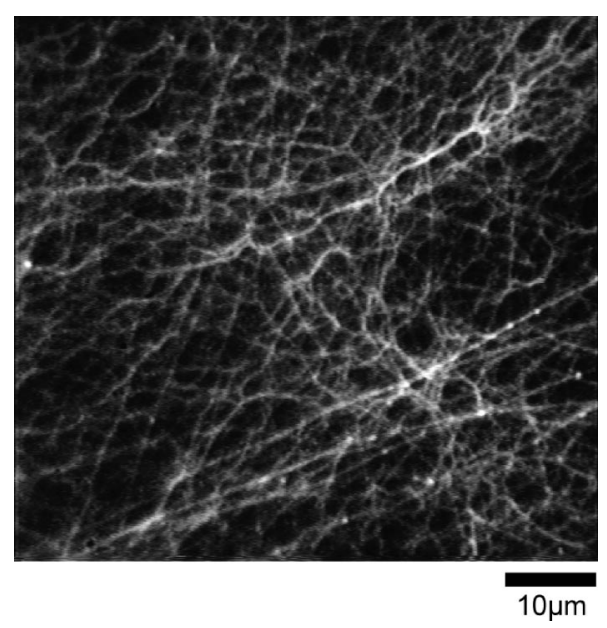

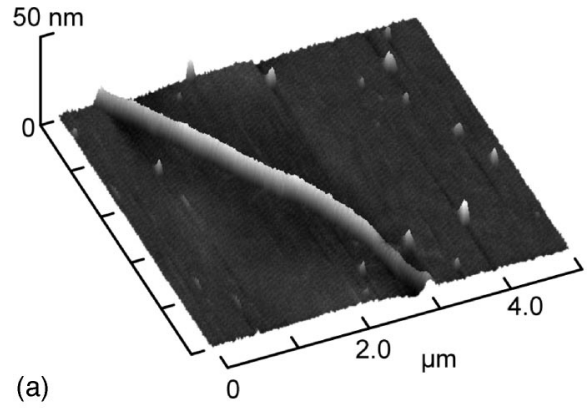

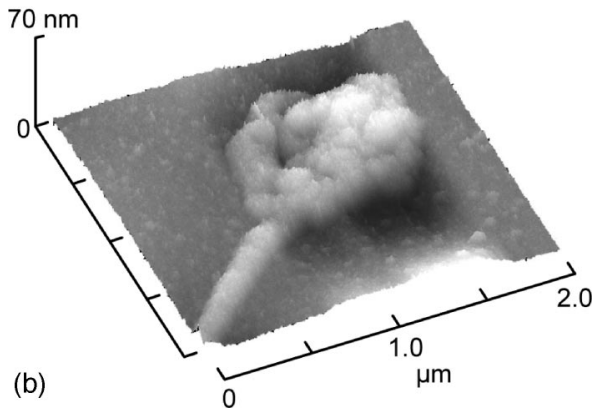

FIG. 4. AFM images of a DNA bundle adsorbed on a glass surface. (a) Coexistence of a thick bundle and compacted single chains. The width and height of the bundle are 250 and $10 \mathrm{~nm}$, respectively. (b) Structure near the end of a thick bundle.

characteristics, i.e., the elongated state is observed at a low $\operatorname{SPD}(3+)$ concentration (below 110-130 $\mu \mathrm{M}$ ), a region of coexistence is found up to $170-180 \mu \mathrm{M}$, and a "condensed" state, consisting of either single-chain compaction or multiple-chain assembly, is formed at higher concentrations. A significant feature at high DNA concentrations is the formation of thick bundles composed of multiple DNA molecules.

Figure 4 shows typical AFM images measured under a high DNA concentration, corresponding to the top bar in Fig. 3. It is apparent that a thick bundle is formed from the parallel assembly of DNA molecules. The thick bundle is apparently $250 \mathrm{~nm}$ wide and $10 \mathrm{~nm}$ high. Since it is semiflexible or soft, as shown in Fig. 1(b), the thick bundle can be deformed, so that its contact with the glass surface increases. In addition, the apparent width is somewhat larger than the actual size because of an artifact due to the thickness of the AFM tip. Based on the cross-sectional area, we calculated that each thick bundle could contain as many as 600 DNA segments, if the bundle is tightly packed with doublestranded DNAs with a diameter of $2 \mathrm{~nm}$. However, the rather large fluctuational motion of the bundle, as seen in Fig. 1(b), suggests that the packing is not so tight. Thus the actual number of DNA segments across the cross section should be somewhat less than 600 . In addition, the end of the bundle shows a quasispherical structure, as seen in Fig. 4. Since individual T4 DNA molecules have a full length of approximately $57 \mu \mathrm{m}^{3}$, long DNA chains are expected to bend back on their ends, at least for bundles with lengths of several $\mu \mathrm{m}$, as in Fig. 1. In addition to the bundle, individual DNA molecules in folded compact states are also observed in the upper panel in Fig. 4. This coexistence of bundle structures with multiple chains and a compact state with single
IG. 5. Network structure formed from a lot of DNA molecules. A small droplet $(40 \mu \mathrm{L})$ of $10 \mu \mathrm{M}$ T4 DNA solution labeled by $0.1 \mu \mathrm{M}$ DAPI was placed on a glass plate, and then equal volume of $1 \mathrm{mM} \mathrm{SPD}(3+)$ solution was transferred on the plate so as to contact with the T4 DNA solution. Fluorescence microscopic image on the glass plate, near the contact line between the solution of DNA and $\operatorname{SPD}(3+)$, is shown.

chains corresponds well to the observations by fluorescence microscopy (Fig. 1).

Figure 5 shows the network structure generated by mixing DNA solution with a large excess of the condensing agent spermidine. When the effective attractive interaction between DNA segments is very strong, as in this case, it is difficult for DNA molecules to find the optimal arrangement during thermal agitation. Thus the DNA molecules are kinetically "frozen" into a network structure, as in Fig. 5. A molecular-dynamics study has shown the spontaneous formation of oriented bundles in a model stiff polyelectrolyte system through a certain kinetic process. $^{23}$

\section{NUMERICAL SIMULATION}

An off-lattice Monte Carlo simulation was performed to compare the collapse transitions in very dilute and semidilute solutions of semiflexible polymer chains. We describe our polymer chain as a sequence of $N_{m}$ spherical monomers of diameter $d$ connected by elastic bonds in three-dimensional space. The number of polymer chains is $N_{p}$, which are placed in a cubic periodic box with a box length of $100 \mathrm{~d}$.

The bonding interaction between two successive monomers is treated as being harmonic with cutoffs,

$U_{\text {bond }}(l)= \begin{cases}k_{\text {bond }}(l-d)^{2}, & \text { for } l_{\min }<l<l_{\max } \\ \infty, & \text { for } l \leqslant l_{\min } \text { or } l \geqslant l_{\max },\end{cases}$

where $l$ is the bond length. We set $k_{\text {bond }}=20 k_{B} T, l_{\text {min }}$ $=0.85 d$, and $l_{\max }=1.15 d$, respectively. Chain stiffness is introduced by the bending potential depending on the angle $\theta$ between successive bond vectors,

$$
U_{\text {bend }}(\theta)=k_{\text {bend }}(1-\cos \theta)^{2},
$$

where we set $k_{\text {bend }}=60 k_{B} T$, for which the persistence length of the chain is calculated to be about $14.7 d$. The interaction between DNA segments is modulated by the presence of multivalent cations, but it is very difficult to simulate such a 
(A)

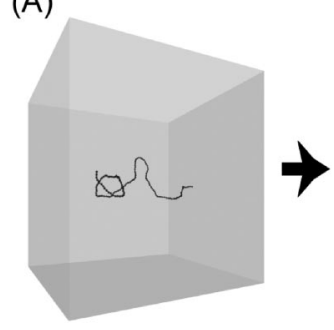

(B)

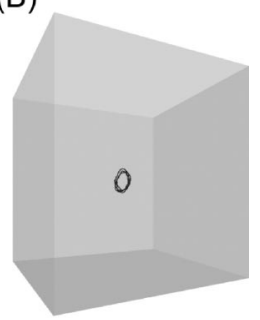

(C)

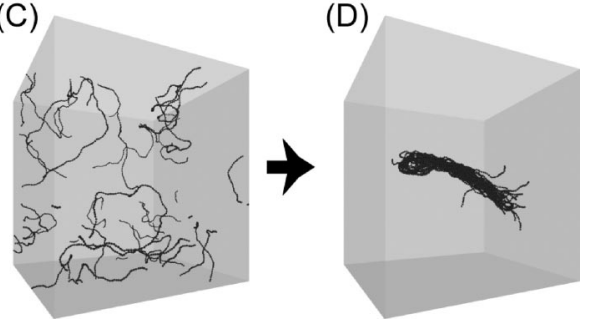

FIG. 6. Snapshots of the change in the conformation of polymer chain(s) using a Monte Carlo simulation. Top: (a) A swollen coil at $\xi / k_{B} T=0.6$; (b) a collapsed toroidal structure at $\xi / k_{B} T=0.63$ in dilute solution $\left(N_{p}=1\right)$. Bottom: (c) Swollen coils at $\xi / k_{B} T=0.38$; (d) a bundle structure composed of multiple-chain assemblies at $\xi / k_{B} T=0.42$ in semidilute solution (number of polymer chains: $N_{p}=14$ ).

realistic system composed of a semidilute long-DNA solution with many multivalent and simple salt ions. Therefore, in this study, we adopted the Lennard-Jones potential to model the effective interaction between nonbonded monomers:

$$
U_{L-J}(r)= \begin{cases}\left.4 \xi\left(\frac{d}{r}\right)^{12}-\left(\frac{d}{r}\right)^{6}-c\right], & \text { for } r \leqslant R_{c} \\ 0, & \text { for } r \geqslant R_{c}\end{cases}
$$

where $r$ is the distance between two monomers, $\xi$ controls the strength of the interaction and $c=\left(d / R_{c}\right)^{12}-\left(d / R_{c}\right)^{6}$ so that the potential value becomes zero at the cutoff $R_{c}$ $=2.5 \mathrm{~d}$. Considering that multivalent ion-mediated attraction has a rather short range, while long-range Coulomb repulsion is well screened under the usual experimental conditions, such a simplified model with a Lennard-Jones potential may be a good tool for examining some aspects of DNA collapse, as has been demonstrated previously. ${ }^{24,25}$ To sample the configurational space effectively, in addition to the local jumps of monomers, we adapted reptation algorithm, in which one monomer is removed from a randomly selected one chain end and attached to the other end.

Figure 6 shows snapshots of the conformation of polymer(s) with the chain length $N_{m}=150$ from the simulation in very dilute and semidilute solutions, respectively. In very dilute solution $\left(N_{p}=1\right)$, a chain takes a swollen coil conformation [Fig. 6(a)] at small $\xi$. An increase in $\xi$ leads to a marked discontinuous transition or first-order phase transition to the ordered collapsed structure. To minimize the energetic penalty associated with bending, a semiflexible chain, such as giant DNA, wraps itself circumferentially to take a toroidal morphology [Fig. 6(b)] in very dilute solution. Larger $\xi$ corresponds to an experimental condition with a higher multivalent cation concentration. In the semidilute so- lution $\left(N_{p}=14\right)$, we also observed a marked discontinuous transition from a state characterized by overlapping chains with a coiled conformation [Fig. 6(c)] to an ordered densely packed state [Fig. 6(d)] with an increase in $\xi$. In contrast to the very dilute solution, multiple chains form a bundle in the semidilute solution. This is a consequence of the simple fact that making contact with monomers in other chains does not require bending energy. At an intermediate chain concentration $\left(N_{p}=4\right)$, the transition is observed around $\xi / k_{B} T$ $\sim 0.48$, indicating the generation of two different ordered structures as collapsed products from multiple chains: bundle and toroid. We confirmed that the latter is energetically more stable, which corresponds to the theoretical consideration. Thus the appearance of the bundle for $N_{p}=4$ is attributable to a kinetic effect, in other words, the bundle is considered as a metastable state in this condition.

We should note the significant effect of kinetics in the multiple-chain system, i.e., it is rather difficult to attain true thermal equilibrium. In our simulation without the reptation algorithm, we sometimes observed a condition in which bundles with some chains and other small aggregates or collapsed single chains coexist at some stage during the collapse. If actual DNAs form such small aggregates or collapsed single chains during the collapsing process experimentally, they may behave like charged colloidal particles, which would enable such small objects and the bundle to coexist.

\section{DISCUSSION}

\section{Switching of events between single and multiple chains}

DNA molecules in viral capsids, bacterial nucleotides, and nuclei of eukaryotes occupy a volume $10^{-4}-10^{-6}$ times as small as that when they exist free in aqueous solution. While living matter has an elaborate apparatus for packing DNA, a similar dramatic decrease in volume to a folded compact state can be observed in vitro simply by adding various kinds of chemical agents, such as polyamines, ${ }^{14-17}$ multivalent metal cations, ${ }^{26-28}$ neutral polymer, ${ }^{29-31}$ cationic polymer, ${ }^{32,33}$ cationic surfactant, ${ }^{34-36}$ or alcohol. ${ }^{37-39}$ Extensive studies on such drastic changes in DNA have been carried out. Most of these past studies have dealt with "DNA condensation" without any clear distinction between the events involving single DNA molecules and multiple DNA molecules, i.e., between the folding transition of single chains and aggregation (or precipitation). Under these conditions, it has been stated that "use of the term condensation is generally confined to situations in which the aggregate is of finite size and orderly morphology." ${ }^{40}$ In the literature, "DNA condensation" has frequently been considered a highly cooperative phenomenon, where the transition was regarded to be steep but continuous.

In contrast, using the experimental technique of singlechain observation, it has recently been established that the transition is all-or-none at the level of individual single giant DNAs. ${ }^{4-6}$ Figure 1 shows such a discrete transition for individual T4 DNA molecules. Thus, one of the main purposes of this study was to clarify the distinction between the com- 
paction of single DNA and the condensation of multiple DNAs.

It is known that condensation induced by multivalent cations generally gives particles with characteristic morphologies, such as a toroid and a rod. It has been reported that toroidal particles, with circumferentially wrapped DNA and rather uniform in size (outer diameter of $60-100 \mathrm{~nm}$ ) regardless of the DNA source, were most commonly generated with spermidine, ${ }^{14-16}$ spermine, ${ }^{15}$ or $\mathrm{Co}\left(\mathrm{NH}_{3}\right)_{6}^{3+}$ (Refs. $26,28)$ as a condensing agent, while permethylated spermidine produced a high proportion of rods. ${ }^{41}$ It has also been reported that rods, as well as fibers, were found at high ethanol concentrations. ${ }^{42}$ Since the width of these rods and fibers was $10-30 \mathrm{~nm}$, the cross-sectional area is much smaller than that of the bundle reported in the present study. The formation of thin rods and fibers has been attributed to the structural transition of DNA from B to A form at a high alcohol concentration. Thus it may be reasonable to conclude that the rods and fibers are quite different from the thick bundle. With regard to bundle formation, a recent theoretical study $^{43}$ on columnar DNA assemblies suggested a variety of order phases and a bundling transition.

It has become clear that a single semiflexible polymer undergoes a folding transition from an elongated coil state to a compact state with a morphology such as a toroid or rod, based on a systematic study of the folding transition of single giant DNA molecules together with theoretical analyses. The ratio of the toroid and rod forms is determined as a function of both the stiffness of the molecular chain and the effective attraction between DNA segments. ${ }^{24}$

The present results indicate the spontaneous formation of a thick bundle, besides a toroid and a rod, with an increase in the DNA concentration. It has been reported that other semiflexible polymers, such as actin ${ }^{44}$ and filamentous bacteriophages fd and M13, ${ }^{45}$ form bundles spontaneously. The mechanism of bundle formation in these charged semiflexible polymers is expected to be essentially the same as that described here. In addition, the critical concentration of $\operatorname{SPD}(3+)$ needed to induce a thick bundle at a high DNA concentration is essentially the same as that needed to generate single DNA compaction at a low DNA concentration. This may be important because the polyamine concentration is considered an intensive variable, or an environmental parameter. $^{46,47}$

\section{Theoretical consideration of relative stability}

Let us consider a polymer solution with a concentration below or around the overlap threshold. We divide the whole system into many subsystems of volume $\Omega$, which is small but still much larger than the volume occupied by a single chain. If we take a snapshot at some moment, the number of chains in the $i$ th subsystem, $N_{i}$, should show considerable deviation from the average due to strong spatial inhomogeneity. In addition, the diffusion coefficient of the chain is low, especially for a long chain. Thus by neglecting the flux of the chain through the boundary, we tentatively regard the subsystem as the canonical ensemble (this corresponds to the model we adopted in the simulation). When an adequate amount of condensing agent is added to the system at $t=0$, each subsystem may be driven to equilibrium. Depending on $N_{i}$ at $t=0$, the equilibrium state corresponds to the dispersed toroidal particles with a single- or multichain assembly. Let us consider the energetic term for a toroid made from a semiflexible chain with segment diameter $d$, persistence length $\lambda$, and total contour length $L$. The geometry of the toroid is characterized by its average radius $R$ and the cross-sectional radius $r$. The volume energy is written as

$$
U_{v o l} \approx-\varepsilon d^{2} L,
$$

where $\varepsilon$ is the cohesion energy density. By denoting the surface tension $\sigma=\varepsilon d$ and the bending modulus $\kappa=k_{B} T \lambda$, the surface energy and bending energy are

$$
\begin{aligned}
& U_{\text {sur }} \approx \sigma r R, \\
& U_{\text {bend }} \approx \kappa \frac{L}{R^{2}} .
\end{aligned}
$$

The optimization of $U_{\text {sur }}+U_{\text {bend }}$ under the constraint $R r^{2}$ $=d^{2} L$ leads to the optimum size ${ }^{48}$

$$
R \approx\left(\frac{L \kappa^{2}}{d^{2} \sigma^{2}}\right)^{1 / 5} \text {. }
$$

Thus, taking the translational freedom of $N_{i}$ toroidal particles into account, the free energy of the dispersed toroidal particles in asymptotically dilute solution is

$F_{\text {toroid }}^{1} \approx-\varepsilon d^{2} L N_{i}+(\sigma d)^{4 / 5} \kappa^{1 / 5} L^{3 / 5} N_{i}+N_{i} \ln \left(\frac{N_{i}}{\Omega}\right)$.

When the number of chains in the subsystem $N_{i}$ increases, they may form a toroidal assembly or bundle. The free energy of the toroidal assembly and bundle with $N_{i}$ chains are

$$
\begin{aligned}
& F_{\text {toroid }} \approx-\varepsilon d^{2} L N_{i}+(\sigma d)^{4 / 5} \kappa^{1 / 5}\left(L N_{i}\right)^{3 / 5}, \\
& F_{\text {bundle }} \approx-\varepsilon d^{2} L N_{i}+\sigma d L N_{i}^{1 / 2},
\end{aligned}
$$

where we neglect the end effect of the bundle.

Analysis of the above three free-energy expressions shows that single-chain collapse occurs in the very dilute region, whereas a bundle is formed in the rather concentrated region. The toroidal assembly with multiple chains may be formed at an intermediate concentration, which is in excellent agreement with the simulation. There are two important time scales in the system under consideration: the lifetime of the metastable coil $t_{c}$ and the characteristic amount of time needed for the chain to diffuse through a subsystem of size $t_{d}$. Since it is possible to set the size of the subsystem to satisfy the relationship $t_{c}<t_{d}$, the above analysis makes sense. Once single-chains collapse into the toroid, or small assemblies are formed, it is practically impossible to achieve true equilibrium due to the large activation barrier. The chains do not aggregate further, but rather coexist as a result of their similarity to charged colloidal particles.

\section{Why does the DNA bundle have a finite thickness?}

For simplicity, we consider a bundle of radius $a$ composed of $n$ polymer chains (e.g., Fig. 7). When $L \gg a$, the 


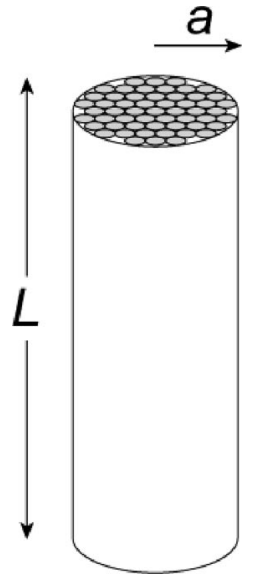

FIG. 7. Schematic representation of a thick bundle. $L$ and $a$ indicate the length and radius of a thick bundle, respectively.

electrostatic energy of a bundle, with surface charge density $\rho_{s}$ and no charge in the volume part, is calculated per unit length as

$$
U_{\text {surf }} \approx \frac{\rho_{s}^{2} a^{2}}{\varepsilon_{2}} \ln \frac{a+\lambda_{D}}{a},
$$

where $\varepsilon_{2}$ and $\lambda_{D}$ are the dielectric constant of the solvent and Debye length. The volume energy in the bundle should be proportional to the cross-sectional area, $\pi a^{2}$, or to the number of polymer chains $n$,

$$
U_{i n t} \approx-\alpha \pi a^{2},
$$

where $\alpha$ is a constant that represents the degree of stabilization, or the attractive energy between polymer chains. Since $U_{\text {int }}$ is strongly dependent on $a$, there is no critical value of $a$ for inducing destabilization of a polymer bundle. The bundle tends to thicken indefinitely, rather than dissolving into individual chains. Next, we consider a bundle with a surviving electric charge in the volume part. When the charge density is given by $\rho_{v}$, the electrostatic energy is given as

$$
U_{v o l} \approx \rho_{v}^{2} a^{4}\left(\frac{1}{4 \varepsilon_{1}}+\frac{1}{\varepsilon_{2}} \ln \frac{a+\lambda_{D}}{a}\right),
$$

where $\varepsilon_{1}$ is the dielectric constant in the volume part of the bundle. It is obvious that $U_{v o l}$ dominates $U_{i n t}$ above a certain value of $a$, i.e., the bundle should be most stable at a finite thickness.

In actual experiments, as in the present study, a kinetic effect should also be considered. ${ }^{49}$ However, the thickness should show a wide distribution when the kinetic effect is dominant. The present experimental trend indicates that the thickness of the DNA bundle is rather uniform. Thus it may be useful to consider the effect of any surviving negative charge to determine the thickness of the bundle.

\section{CONCLUSION}

We have performed the experiments on the conformational transition of giant DNA molecules, by comparing single-chain and multiple-chain events by use of the experimental methodology of single-chain observation. The results are summarized as follows:

(i) Long DNA molecules undergo single-chain compaction at a low DNA concentration, whereas they form a thick bundle at high concentrations. This transition can be interpreted in terms of the first-order phase transition according to the criterion of Landau. ${ }^{50}$

(ii) The critical concentrations of $\operatorname{SPD}(3+)$ needed to induce the folding transition of single DNAs are essentially the same as those needed to generate a thick bundle with multiple DNAs, indicating that this may be an intensive variable, or an environmental parameter of the polyamine.

(iii) The thickness of the bundle is rather uniform. As many as $10^{2}$ segments of DNA may be situated in the cross section of the thick bundle.

(iv) DNA molecules form network structures instead of a bundle when there is rather strong attractive interaction between the segments.

\section{ACKNOWLEDGMENT}

This study was supported in part by a Grant-in-Aid for Scientific Research from the Ministry of Education, Science, Culture, and Sports of Japan.

${ }^{1}$ M. Yanagida, Y. Hiraoka, and I. Katsuya, Cold Spring Harbor Symp. Quant. Biol. 47, 177 (1983).

${ }^{2}$ C. Bustamante, Annu. Rev. Biophys. Biophys. Chem. 20, 415 (1991).

${ }^{3}$ K. Yoshikawa, Y. Matsuzawa, K. Minagawa, M. Doi, and M. Matsumoto, Biochem. Biophys. Res. Commun. 188, 1274 (1992).

${ }^{4}$ K. Yoshikawa and Y. Matsuzawa, Physica D 84, 220 (1995).

${ }^{5}$ K. Yoshikawa, S. Kidoaki, M. Takahashi, V. V. Vasilevskaya, and A. R. Khokhlov, Ber. Bunsenges. Phys. Chem. 100, 876 (1996).

${ }^{6}$ M. Takahashi, K. Yoshikawa, V. V. Vasilevskaya, and A. R. Khokhlov, J. Phys. Chem. B 101, 9396 (1997).

${ }^{7}$ Y. Yamasaki, Y. Tcramoto, and K. Yoshikawa, Biophys. J. 80, 2823 (2001).

${ }^{8}$ V. A. Bloomfield, Curr. Opin. Struct. Biol. 6, 334 (1996).

${ }^{9}$ J. A. Schellman and N. J. Parthasaratary, Mol. Biol. 175, 313 (1984).

${ }^{10}$ J.-L. Sikorav, J. Pelta, and F. Livolant, Biophys. J. 67, 1387 (1994).

${ }^{11}$ J. Pelta, D. Durand, J. Doucet, and F. Livolant, Biophys. J. 71, 48 (1994).

${ }^{12}$ J. Pelta, F. Livolant, and J.-L. Sikorav, J. Biol. Chem. 271, 5656 (1996).

${ }^{13}$ P. G. de Gennes and J. Prost, The Physics of Liquid Crystals, 2nd ed. (Oxford University Press, New York, 1993).

${ }^{14}$ L. C. Gosule and J. A. Schellman, Nature (London) 259, 333 (1976).

${ }^{15}$ D. K. Chattoraj, L. C. Gosule, and J. A. Schellman, J. Mol. Biol. 121, 327 (1978).

${ }^{16}$ K. A. Marx and G. C. Ruben, Nucleic Acids Res. 11, 1839 (1983).

${ }^{17}$ C. B. Post and B. H. Zimm, Biopolymers 21, 2139 (1982).

${ }^{18}$ B. G. Feuerstein, N. Pattabiraman, and L. J. Marton, Proc. Natl. Acad. Sci. U.S.A. 83, 5948 (1986)

${ }^{19}$ I. S. Haworth, A. Rodger, and W. G. Richards, J. Biomol. Struct. Dyn. 10, 195 (1992).

${ }^{20}$ A. P. Lyubartsev and L. Nordenskiöld, J. Phys. Chem. B 101, 4335 (1997).

${ }^{21}$ N. Korolev, A. P. Lyubartsev, L. Nordenskiöld, and A. Laaksonen, J. Mol. Biol. 308, 907 (2001).

${ }^{22}$ N. Yoshinaga, T. Akitaya, and K. Yoshikawa, Biochem. Biophys. Res. Commun. 286, 264 (2001).

${ }^{23}$ M. J. Stevens, Phys. Rev. Lett. 82, 101 (1999).

${ }^{24}$ H. Noguchi and K. Yoshikawa, J. Chem. Phys. 109, 5070 (1998).

${ }^{25}$ T. Sakaue and K. Yoshikawa, J. Chem. Phys. 117, 6323 (2002).

${ }^{26}$ J. Widom and R. L. Baldwin, J. Mol. Biol. 144, 431 (1980).

${ }^{27}$ J. Widom and R. L. Baldwin, Biopolymers 22, 1595 (1983).

${ }^{28}$ H. Deng and V. A. Bloomfield, Biophys. J. 77, 1556 (1999).

${ }^{29}$ L. S. Lerman, Proc. Natl. Acad. Sci. U.S.A. 68, 1886 (1971). 
${ }^{30}$ Yu. M. Evdokimov, A. L. Platonov, A. S. Tikhonenko, and Ya. M. Varshavskii, FEBS Lett. 23, 180 (1972).

${ }^{31}$ U. K. Laemmli, Proc. Natl. Acad. Sci. U.S.A. 72, 4288 (1975).

${ }^{32}$ K. Yoshikawa, Y. Yoshikawa, Y. Koyama, and T. Kanbe, J. Am. Chem. Soc. 119, 6473 (1997).

${ }^{33}$ A. L. Martin, B. J. Davies, B. J. Rackstraw, C. J. Roberts, S. Stolnik, S. J. B. Tendler, and P. M. Williams, FEBS Lett. 480, 106 (2000).

${ }^{34}$ K. Hayakawa, J. P. Santerre, and J. C. T. Kwak, Biophys. Chem. 17, 175 (1983).

${ }^{35}$ K. Shirahama, K. Takashima, and N. Takisawa, Bull. Chem. Soc. Jpn. 60, 43 (1987).

${ }^{36}$ K. Shirakawa, T. Masaki, and K. Takashima, in Microdomains in Polymer Solutions, edited by P. Dubin (Plenum, New York, 1985), p. 299.

${ }^{37}$ E. P. Geiduschek and I. Gray, J. Am. Chem. Soc. 78, 879 (1956).

${ }^{38}$ D. Lang, J. Mol. Biol. 46, 209 (1969).

${ }^{39}$ T. H. Eickbush and E. N. Moudrianakis, Cell 13, 295 (1976).

${ }^{40}$ V. A. Bloomfield, Biopolymers 44, 269 (1997).

${ }^{41}$ G. E. Plum, P. G. Arscott, and V. A. Bloomfield, Biopolymers 30, 631 (1990).
${ }^{42}$ P. G. Arscott, C. Ma, J. R. Wenner, and V. A. Bloomfield, Biopolymers 36, 345 (1995).

${ }^{43}$ H. M. Harreis, A. A. Kornyshev, C. N. Licos, H. Löwen, and G. Sutmann, Phys. Rev. Lett. 89, 018303 (2000).

${ }^{44}$ J. X. Tang, S. Wong, P. T. Tran, and P. A. Janmey, Ber. Bunsenges. Phys. Chem. 100, 796 (1996).

${ }^{45}$ J. X. Tang, P. A. Janmey, A. Lyubartsev, and L. Nordenskiöld, Biophys. J. 83, 566 (2002).

${ }^{46}$ K. Yoshikawa, J. Biol. Phys. 28, 701 (2002).

${ }^{47} \mathrm{~K}$. Yoshikawa and Y. Yoshikawa, in Pharmaceutical Perspectives of Nucleic Acid-Based Therapeutics, edited by R. I. Mahato and S. W. Kim (Taylor \& Francis, London, 2002), p. 136.

${ }^{48}$ A. Yu. Grosberg and A. V. Zhestkov, J. Biomol. Struct. Dyn. 3, 859 (1986).

${ }^{49}$ B.-Y. Ha and A. J. Liu, Phys. Rev. Lett. 81, 1011 (1998).

${ }^{50}$ L. D. Landau and E. M. Lifshits, Statistical Physics (Elsevier, New York, 1994). 